\title{
Revisão integrativa sobre a infecção pelo vírus da imunodeficiência humana em idosos
}

\author{
Integrative review of human immunodeficiency virus infection in the elderly
}

\author{
Revisión integral de la infección por el virus de la inmunodeficiencia humana en los \\ ancianos
}

Amanda Cordeiro da Silva Arruda1*, Diogenes José Gusmão Coutinho.

\begin{abstract}
RESUMO
Objetivo: Analisar a relação HIV X terceira idade. Métodos: Trata-se de um estudo com abordagem exploratória, na qual utilizou-se como procedimento técnico o levantamento bibliográfico, na base de dados da Scientific Electronic Library Online (SciELO), Literatura Latino-Americana e Caribenha em Ciências da Saúde (LILACS) e Coordenação de Aperfeiçoamento de Pessoal de Nível Superior (CAPES). Resultados: Foram analisados onze (11) artigos, culminando em uma reflexão e diálogo acerca dos assuntos abordados e as ideias trazidas evidenciaram que a pessoa com HIV ajusta-se negativamente frente às dificuldades sexuais, sociais e emocionais; o estigma e a descriminação relacionados a vida sexual a que estão submetidas pessoas portadoras do vírus da imunodeficiência humana, acarreta o aumento da incidência de HIV/AIDS na população acima de 50 anos, necessitando que os profissionais de saúde enxerguem que pacientes idosos estão propícios a infecção pelo vírus HIV. Considerações finais: A análise possibilitou constatar que, os tratamentos para portadores da imunodeficiência humana têm obtido êxito o que contribuiu para um número crescente de idosos portadores do HIV/AIDS em virtude da nova expectativa de vida, todavia, existem muitos tabus no que se refere a sexualidade na terceira idade, necessitando dar-lhes maior atenção e esclarecimento.
\end{abstract}

Palavras-chave: Terceira idade, Sexualidade, HIV, AIDS.

\begin{abstract}
Objective: To analyze the relationship between HIV and the elderly people. Methods: It's about a study with an exploratory approach, in which the bibliographic survey was used as a technical procedure, in the database of the Scientific Electronic Library Online (SciELO), Latin American and Caribbean Literature in Health Sciences (LILACS ) and Coordination for the Improvement of Higher Education Personnel (CAPES). Results: Eleven (11) articles were analyzed, culminating in a reflection and dialogue about the subjects covered and the ideas brought up showed that the person with HIV adjusts negatively in the face of sexual, social and emotional difficulties; the stigma and discrimination related to sexual life to which people with the human immunodeficiency virus are subjected, leads to an increase in the incidence of HIV / AIDS in the population over 50 years old, requiring health professionals to see that elderly patients are prone to HIV virus infection. Final considerations: The analysis made it possible to verify that the treatments for people with human immunodeficiency have been successful, which contributed to an increasing number of elderly people with HIV / AIDS due to the new life expectancy, however, there are many taboos regarding sexuality in old age, needing to give them more attention and clarification.
\end{abstract}

Keywords: Third age, Sexuality, HIV, AIDS.

${ }^{1}$ Faculdade Alpha, Recife - PE. *E-mail: cordeiroamanda2020@gmail.com 


\section{RESUMEN}

Objetivos: Hacer una análise, la relación HIV X tercera edad. Métodos: Se trata de un estudio con abordagen de exploración, en cual se utilizó como procedimiento técnico el levantamiento bibliográfico en la base de datos de la Biblioteca Científica Electrónica en Línea (SciELO), Literatura Latinoamericana y del Caribe en Ciencias de la Salud (LILACS) y Coordinación de Perfeccionamiento de Personal de Educación Superior (CAPES). Resultados: Fueran analisados once (11) artículos culminando en una reflexión y charla cerca de los contenidos abordados la ideas traedas demostró en su estudio que las personas con VIH se ajusta negativamente ante las dificultades sexuales, sociales y emocionales; el estigma y la discriminación relacionados con la vida sexual a la que están sometidas las personas con el virus de la inmunodeficiencia humana, conduce a un aumento en la incidencia del VIH / SIDA en la población mayor de 50 años, lo que obliga a los profesionales de la salud a ver que los pacientes de edad avanzada son propensos al la infecction por VIH. Consideraciones finales: El análisis mostró que los tratamientos para personas con inmunodeficiencia humana han tenido éxito, lo que ha contribuido a que un número creciente de personas mayores con VIH / SIDA debido a la nueva esperanza de vida, sin embargo, existen muchos tabúes con respecto a la sexualidad en ancianos, que necesitan darles más atención y aclaración.

Palabras clave: Tercera edad, Sexualidad, HIV, AIDS.

\section{INTRODUÇÃO}

A infecção pelo Vírus da Imunodeficiência Humana - HIV vem ganhando destaque na pauta das discussões no ambiente nacional e internacional. Isso se explica através da compreensão e do entendimento por parte das autoridades e população, de que a saúde pública é um dos fatores determinantes para o bom desenvolvimento de um país (MILZ T, 2019).

O HIV é caracterizado por uma doença infecciosa provocada por vírus do gênero Lentivirus e família Retroviridae, que ataca o sistema imunológico, responsável por defender o organismo de diversas doenças. O vírus pode ser transmitido pelo contato sanguíneo, pela transmissão de mãe para filho ou via sexual através da penetrabilidade na mucosa vaginal e anal (BRASIL, 1999).

A disponibilidade de Terapia Antirretroviral Altamente Ativa (HAART) desde meados da década de 90 aumentou significativamente a expectativa de vida das pessoas vivendo com HIV. A cobertura mundial da terapia antirretroviral (TARV) aumentou de 7\% em 2003 para 42\% em 2008. Nesta perspectiva, aproximadamente 12 milhões de anos de vida foram adicionados ao mundo entre 1996 e 2008 como resultado do amplo acesso à HAART (UNAIDS, 2020).

De acordo com dados do Boletim Epidemiológico HIV/Aids no primeiro semestre de 2019 foi notificado no Brasil 300.496 casos de infecção pelo HIV, um crescimento de 583,62\% se comparado ao ano anterior. É importante ressaltar que a região sudeste do país possui o maior número de notificações nos anos supracitados (BRASIL, 2019).

Quando abordado o HIV na terceira idade, necessário se faz difundir a definição de pessoa idosa comumente utilizada pela Organização Mundial de Saúde (OMS) e médicos geriatras, os quais são indivíduos com 60 anos ou mais, o que difere da conceituação atribuída a indivíduos "idosos" aqueles com 50 anos ou mais de acordo com o Centro de Controle e Prevenção de Doenças (CDC) dos EUA. Para este artigo utilizaremos a concepção apresentada pelo CDC (MACIEL MG, 2010).

Exposto tais conceituações, faz-se necessário salientar que, o Brasil tem uma das populações que mais envelhece no mundo e a expectativa de vida alcançou 76,3 anos em 2018 (IBGE, 2018). E de acordo com dados do Boletim Epidemiológico HIV/Aids (2019), os casos de indivíduos idosos portadores do vírus HIV são para os homens de $37,1 \%$ ( 50 a 54 anos), 23,6\% (55 a 59 anos) e 12,4\% (60 anos ou mais); já as mulheres somam $16,1 \%$ (50 a 54 anos), $12,7 \%$ (55 a 59 anos) e 5,7\% (60 anos ou mais), o que pode ser justificado pelo fato de indivíduos mais velhos terem menor tendência a praticar sexo seguro, aumentando o risco de infecção pelo vírus (BRASIL, 2019). 
Esse cenário apresenta alguns desafios, incluindo o fato de que o diagnóstico de HIV tende a atrasar em idosos e alguns sintomas podem ser confundidos com outras doenças comuns para a idade, não sendo considerados de alto risco para infecção pelo HIV de acordo com os profissionais de saúde. Vale salientar que, em indivíduos já diagnosticados com HIV, apresenta-se o desafio de morbidade associada ao envelhecimento, como também o fato de pacientes idosos apresentarem maior suscetibilidade aos efeitos tóxicos e interações farmacológicas dos medicamentos, sendo a idade menor de 50 anos uma das indicações para iniciar a HAART de acordo com a diretriz brasileira para TARV em adultos (ROSSI SMG, et al. 2012).

Todavia, vivemos em um século onde a informação chega até nossa residência, seja por meio de livros digitais, impressos e/ou sites de informação e comunicação. Desta feita, conhecer e discutir acerca do crescimento e controle do HIV na terceira idade é de suma importância para novos diagnósticos, tratamento e aumento da sobrevida (ROSSI SMG, et al. 2012).

Assim, o problema que conduziu esta investigação baseou-se na seguinte indagação: De que forma a literatura científica aborda a relação HIV X Terceira idade? Considera-se que, a resposta para esta indagação contribuirá para gerar novos conhecimentos científicos a respeito do tema investigado norteando a construção deste artigo, tendo como objetivo principal analisar a relação HIV X Terceira idade.

\section{MÉTODOS}

Este artigo se classifica quanto aos objetivos do estudo em exploratória, cujo procedimento técnico utilizado foi o levantamento bibliográfico (GIL AC, 2002). A pesquisa bibliográfica possibilita a construção e análise de documentos, livros e diferentes textos, com base em referências teóricas já publicadas (FONSECA JJS, 2002).

Sendo assim, buscou-se artigos na base de dados da Scientific Electronic Library Online (SciELO), Literatura Latino-Americana e Caribenha em Ciências da Saúde (LILACS) e Coordenação de Aperfeiçoamento de Pessoal de Nível Superior (CAPES). Na busca realizada utilizou-se a associação das seguintes palavras-chave: "HIV" AND "Terceira idade" AND "idosos", de modo a responder ao longo da análise a problemática em questão: De que forma a literatura científica aborda a relação HIV X Terceira idade?

Convém salientar que, a abordagem por meio do levantamento bibliográfico possibilitou obter uma visão mais clara do problema e consequentemente aprimoramento deste artigo.

\section{RESULTADOS}

Localizou-se duzentos e vinte e quatro (224) artigos, publicados nos últimos 10 anos (2010 a 2020), destes, vinte e dois (22) foram excluídos pois não estavam disponíveis na íntegra, treze (13) não foram redigidos em Língua Portuguesa e cento e oitenta e quatro (184) não tratava do objeto de estudo desta pesquisa, culminando na análise de cinco (5) artigos, a saber (Quadro 1):

Quadro 1 - Principais obras pesquisadas para a construção deste artigo.

\begin{tabular}{|c|c|l|}
\hline $\begin{array}{c}\text { Ano de } \\
\text { publicação }\end{array}$ & Autores & \multicolumn{1}{c|}{ Título /Ano } \\
\hline 2011 & BATISTA AFO, et.al. & $\begin{array}{l}\text { Idosos: Associação entre o conhecimento da AIDS, atividade } \\
\text { sexual e condições sociodemográficas }\end{array}$ \\
\hline 2012 & DRIEMEIER M, et. al. & $\begin{array}{l}\text { Vulnerabilidade à AIDS entre idosos em um centro urbano da } \\
\text { região central do Brasil }\end{array}$ \\
\hline 2012 & GARCIA GS, et. al. & $\begin{array}{l}\text { Vulnerabilidade dos idosos frente ao HIV/aids: tendências da } \\
\text { produção científica atual no Brasil }\end{array}$ \\
\hline 2012 & MELO HMA, et. al. & $\begin{array}{l}\text { O conhecimento sobre aids de homens idosos e adultos jovens: } \\
\text { um estudo sobre a percepção desta doença }\end{array}$ \\
\hline 2015 & SMIT M, et. al. & $\begin{array}{l}\text { Desafios futuros para o atendimento clínico de uma população } \\
\text { idosa infectada pelo HIV: um estudo de modelagem }\end{array}$ \\
\hline
\end{tabular}

Fonte: Arruda ACS e Coutinho DJE, 2020.

Após a seleção do material, realizou-se a leitura exploratória; e análise dos textos, culminando em uma reflexão crítica acerca dos assuntos abordados. 


\section{DISCUSSÃO}

\section{Efeitos do envelhecimento e da infecção pelo HIV no sistema imunológico}

O timo é um órgão importante envolvido no desenvolvimento do sistema imunológico humano e serve como local primário para a maturação dos linfócitos $T$. Estes são geneticamente diversos e funcionam como células $T$ ingênuas que respondem a novas exposições antigênicas ou como células $T$ de memória que respondem a antígenos aos quais o corpo foi exposto anteriormente (ALMEIDA J e LOURENÇO ML, 2009).

Em particular, a ativação de células T CD4 + auxiliares desencadeia uma resposta imune através da diferenciação e proliferação de células $\mathrm{T}$; ativação de células $\mathrm{B}$, resultando no desenvolvimento e secreção de anticorpos; estimulação de outras células efetoras, como células T citotóxicas CD8 + e macrófagos, através da liberação de citocinas; e/ou hipersensibilidade do tipo retardado (SILVA LC, 2013).

À medida que a pessoa envelhece, ocorre a involução do timo e os volumes tímicos são significativamente menores em pessoas com 45 anos ou mais em comparação com pessoas mais jovens. Além disso, a produção de células $T$ ingênuas diminui com o aumento da idade e a produção tímica é mínima apenas após os 55 anos (GUIMARÃES R e FERRAZ AF, 2002).

O aumento da idade está associado à diminuição da funcionalidade das células $T$, redução da população de células $T$ com memória e menor número de células T citotóxicas CD8 + funcionando corretamente. Esses fatores podem explicar por que as pessoas idosas são mais propensas a novas infecções, demonstram resposta abaixo do ideal às imunizações ou manifestam anergia a testes cutâneos, como o Derivado de Proteína Purificada - PPD (CASAES NRR, 2007).

Além disso, a função tímica e a produção de células $T$ ingênuas podem ser inibidas pela infecção pelo HIV. Como essas alterações no sistema imunológico são muito semelhantes aos efeitos do envelhecimento, a progressão da infecção pelo HIV em idosos pode ser mais acentuada. Não apenas as contagens de células CD4 são significativamente mais baixas em adultos jovens e idosos infectados pelo HIV quando comparadas aos controles com a mesma faixa etária, mas os indivíduos mais velhos infectados pelo HIV têm as contagens mais baixas (MONTEIRO JFA e FIGUEIREDO MAC, 2009).

Em indivíduos mais velhos infectados ou não pelo HIV, a proporção de células T CD8 + funcionais foi menor em comparação aos indivíduos mais jovens, mas os indivíduos mais velhos infectados pelo HIV pareceram ser os mais afetados por terem a menor proporção entre os quatro grupos de comparação (PARKER R e AGGLETON P, 2001).

Como as células T citotóxicas CD8 + são importantes na contenção da replicação do HIV, a redução em seu número e funcionalidade associada à infecção pelo HIV e ao envelhecimento, juntamente com a perda de células CD4, pode ajudar a explicar a progressão acelerada da infecção pelo HIV em adultos mais velhos (CAMARGO CV, et al, 2007).

Finalmente, alterações na expressão do receptor de células $T$ à medida que a pessoa envelhece, como o aumento da expressão do co-receptor CCR5, são importantes na patogênese da infecção pelo HIV e também podem influenciar a progressão da doença. Assim, a infecção pelo HIV pode agravar ou ser sinérgica com os efeitos do envelhecimento no sistema imunológico humano (FREITAS MRI, et al, 2000).

Ajustada para os efeitos gerais do envelhecimento na mortalidade, a sobrevida média para aqueles que foram infectados com idades entre 25 e 34 anos foi de 11 anos, em comparação com 6,6 anos e 4,4 anos naqueles que foram infectados, mais velhos, com 55 a 64 anos e 65 anos ou mais, respectivamente (MELO HMA, et. al, 2012).

Os pesquisadores também descobriram que, para cada aumento de 10 anos na idade no momento da infecção pelo HIV, a taxa geral de mortalidade aumenta em $43 \%$. Além da idade na infecção, o tempo desde a infecção foi positivamente correlacionado com o aumento da mortalidade em idosos (45 a 55 anos) que tiveram a maior taxa de mortalidade em comparação com pessoas jovens com menos de 45 anos. A rápida progressão para a AIDS e a diminuição da sobrevida em pacientes idosos infectados pelo HIV também foram confirmadas em coortes francesas e espanholas (DRIEMEIER M, et. al., 2012). 
O Programa Conjunto das Nações Unidas sobre HIV/AIDS (UNAIDS) e a Organização Mundial da Saúde (OMS) estimam que dos 38 milhões de pessoas que vivem com HIV / AIDS no mundo. Dados de países industrializados e conforme relatado pelo UNAIDS refletem principalmente a epidemia de HIV até os 49 anos de idade. No entanto, alguns dados em idosos foram relatados (UNAIDS, 2020).

Nos EUA, aproximadamente um milhão de pessoas estão infectadas pelo HIV, embora cerca de um quarto a um terço não conheça sua infecção pelo HIV. Estima-se também que cerca de 40.000 novas infecções por HIV ocorram a cada ano (GARCIA GS, 2012).

Embora os 50 anos de idade não sejam geralmente usados para identificar pacientes "idosos", esse limite de idade é frequentemente usado pelo CDC nas estatísticas de HIV / AIDS, uma vez que é muito mais antigo que a idade média ( 30 anos) da população/pessoa típica infectada pelo HIV nos EUA (SANTOS AFM, ASSIS M, 2011).

Em suma, o aumento no número total de pessoas com 50 anos ou mais recém-diagnosticadas com HIV ou vivendo com HIV / AIDS pode ser resultado de diagnóstico tardio ou devido à eficácia da terapia potente atualmente disponível para tratar a infecção pelo HIV.

\section{Comportamentos de risco para o HIV}

O HIV é transmitido através do contato sexual, um exemplo são homens que fazem sexo com homens (HSH) ou heterossexual; assim como contato com o sangue, por uso de drogas intravenosas (UDI), picada de agulha ou transfusão de sangue; e perinatalmente da mãe para criança. Nesta perspectiva, os fatores de risco para pessoas com 50 anos ou mais no momento do diagnóstico de HIV foram alterados (SANTOS AFM, ASSIS M, 2011).

Antes da triagem do suprimento sanguíneo, a transfusão sanguínea era a principal via de transmissão para essa faixa etária. Atualmente, $\mathrm{HSH}$ e UDI representam aproximadamente $50 \%$ dos fatores de risco associados à infecção pelo HIV na população idosa (SMIT M, 2015).

Em uma revisão recente dos fatores de risco para o HIV em idosos, verificou-se que os adultos mais velhos se envolvem em uma variedade de atividades sexuais, embora os homens mais velhos tenham sido mais sexualmente ativos do que as mulheres mais velhas (SMIT M, 2015). Uma grande maioria das mulheres mais velhas não tinha conhecimento de risco pessoal e/ou não havia se envolvido em comportamentos de proteção.

Embora as mulheres mais velhas casadas fossem mais sexualmente ativas do que as mulheres solteiras, as mulheres que tinham parceiros eram menos propensas a usar um método contraceptivo de barreira. $O$ uso de preservativo entre as mulheres parece diminuir com a idade, talvez relacionado a não se preocupar com a gravidez ou a percepção de não estar em risco de Doenças Sexualmente Transmissíveis - DST (CLOSS VE e SCHWANKE CHA, 2012).

Algumas barreiras potenciais ao uso de preservativos incluem dificuldades na comunicação entre parceiros, preocupação por falta de confiança e sentimentos que o parceiro masculino controla o uso de preservativos (GARCIA GS, 2012). Batista AFO, et. al (2011) acrescenta outros fatores que podem aumentar o risco de transmissão do HIV entre mulheres mais velhas podem incluir mudanças nos relacionamentos sexuais devido ao divórcio ou morte e alterações da menopausa na mucosa vaginal, aumentando a probabilidade de trauma e doenças sexualmente transmissíveis.

É válido salientar que, embora os medicamentos utilizados para tratar a disfunção erétil em homens mais velhos não demonstrem resultar em aumento da transmissão do HIV em um estudo, isso ainda é uma possibilidade (RISMAN A, 2005).

Atualmente, as histórias sexuais completas de pacientes mais velhos podem não ser obtidas rotineiramente pelos médicos diante o desconforto em relação à sexualidade do paciente, à orientação sexual ou falta de reconhecimento de que a atividade sexual continuada é importante no bem-estar geral. Além disso, os pacientes mais velhos podem não se sentir confortáveis com a divulgação de informações por achar que deveriam ser discretas (ALMEIDA T e LOURENÇO ML, 2009). 


\section{Tratamento de HIV em pacientes idosos}

Atualmente, não existem diretrizes específicas de tratamento que se concentrem no manejo de adultos mais velhos infectados pelo HIV. Contudo, há informações limitadas sobre a eficácia e segurança de regimes antirretrovirais selecionados para pacientes idosos, visto que, mesmo com o início da terapia anti-retroviral, a recuperação de células CD4 pode ser limitada em pacientes idosos infectados pelo HIV (SANTOS AFM e ASSIS M, 2011).

A recuperação limitada de células CD4 em pacientes idosos infectados pelo HIV pode ser devida à diminuição da função tímica associada à idade e, portanto, uma resposta mais lenta, ou possivelmente como resultado de ter uma contagem mais baixa de células CD4 na linha de base (SILVA VXL, 2009).

Por outro lado, existem alguns estudos sugerindo que a contagem de células CD4 aumentam e as respostas virológicas não são diferentes entre pacientes mais velhos e mais jovens infectados pelo HIV (SMIT $M, 2015)$.

Se os idosos infectados pelo HIV estão em maior risco de efeitos colaterais adversos da terapia antiretroviral é menos bem documentado. Os efeitos colaterais dos medicamentos em geral tendem a ser maiores em pacientes mais velhos e podem estar relacionados a declínios associados à idade na função hepática $\mathrm{e}$ renal (CLOSS VE e SCHWANKE CHA, 2012).

A massa hepática, o fluxo sanguíneo e o metabolismo (via quantidade e função da enzima do citocromo P450) diminuem com a idade. Além disso, a diminuição relacionada à idade na massa renal, fluxo sanguíneo, secreção tubular e filtração glomerular pode levar ao acúmulo de drogas e resultar em toxicidade (GARCIA GS, 2012).

Outros fatores, como a polifarmácia, podem contribuir ainda mais para o aumento de eventos adversos. 0 HIV pode diminuir a função renal, infectando especificamente o rim, levando a uma condição conhecida como nefropatia associada ao HIV (HIVAN). É mais provável que o HIVAN ocorra naqueles que são negros, têm AIDS e não estão em tratamento ARV (CAMARGO CV, et al, 2007).

Alterações relacionadas à idade na composição corporal também podem influenciar a farmacocinética dos medicamentos, alterando o volume de distribuição dos medicamentos. A diminuição do peso corporal e da água corporal total pode levar a níveis mais concentrados de drogas no sangue e tecidos, e pode resultar em efeitos e toxicidade aumentados (FREITAS MRI, et al, 2000).

Por outro lado, o aumento da gordura corporal, que atua como depósito de medicamentos lipossolúveis, pode resultar em concentrações séricas reduzidas de medicamentos e, inicialmente, diminuir os efeitos dos medicamentos. Com doses e tempo repetidos, o acúmulo de drogas lipossolúveis na gordura corporal pode levar à toxicidade (PARKER R e AGGLETON P, 2001).

Uma taxa de absorção gastrointestinal mais lenta pode levar ao início tardio dos efeitos do medicamento. Medicamentos com alta ligação às proteínas podem produzir efeitos aprimorados à medida que a pessoa envelhece e as concentrações de proteínas diminuem (MELO HMA, et. al, 2012).

O genótipo do receptor e do transportador de drogas (por exemplo, glicoproteína P, gp-P ou MDR1) também pode afetar profundamente as concentrações de drogas e as respostas ARV resultantes. Alterações na produção ou função da gp-P ou citocromo nos idosos podem levar a respostas alteradas aos medicamentos (MONTEIRO JFA e FIGUEIREDO MAC, 2009).

Uma vez que o Efavirenz exerce frequentemente efeitos no sistema nervoso central, recomenda-se precaução quando utilizado em doentes idosos com demência ou outro distúrbio psiquiátrico ou cognitivo subjacente. O tenofovir está associado à toxicidade renal e deve ser usado com cautela em pacientes com função renal reduzida (ALMEIDA T e LOURENÇO ML, 2009).

Além disso, todos os agentes anti-retrovirais podem potencialmente causar hepatotoxicidade, especialmente NNRTIs e IPs, e podem aumentar o risco de efeitos hepáticos adversos em pacientes com doença hepática subjacente ou insuficiência hepática (CAMARGO CV, et al, 2007). 
Para limitar ainda mais a toxicidade, todos os NRTIs (com exceção do Abacavir) devem ser ajustados na dose na insuficiência renal. Deve-se notar que dados limitados estão disponíveis para fornecer orientação sobre ajustes posológicos específicos de outros anti-retrovirais quando utilizados especificamente em pacientes idosos com insuficiência renal e/ou hepática (FREITAS MRI, et al, 2000).

Comparados às pessoas não infectadas, os pacientes infectados pelo HIV parecem ter uma maior prevalência de síndrome metabólica. A terapia com HAART também tem sido implicada em causar uma síndrome metabólica caracterizada por diminuição da tolerância à glicose e aumento da resistência à insulina, dislipidemias, pressão arterial elevada e obesidade abdominal, condições que parecem estar principalmente associadas ao uso a longo prazo de inibidores da protease do HIV (ALMEIDA T e LOURENÇO LM, 2009).

Essas alterações metabólicas induzidas por medicamentos, juntamente com a idade avançada, têm sido associadas a um risco aumentado de doença cardiovascular e infarto do miocárdio (CASAES NRR, 2007).

Dados limitados até o momento sugerem que pacientes mais velhos infectados pelo HIV experimentam mais eventos adversos e correm maior risco de anormalidades laboratoriais do que pacientes mais jovens infectados pelo HIV recebendo terapia ARV (SMIT M, 2015).

O curso da epidemia de HIV mudou dramaticamente nas últimas três décadas, em grande parte como resultado da crescente conscientização, implementação de estratégias de prevenção e disponibilidade de terapia antirretroviral potente, especialmente nos países desenvolvidos. No entanto, os dados epidemiológicos atuais mostram mudanças na demografia da população HIV (MONTEIRO JFA e FIGUEIREDO MAC, 2009).

À medida que a população de HIV envelhece e a taxa de infecções recém-detectadas em idosos aumenta, os médicos devem estar cientes da crescente necessidade de equilibrar os cuidados com o HIV e o manejo de condições comórbidas comumente associadas ao envelhecimento. Até o momento, não há diretrizes disponíveis que abordem especificamente as necessidades do paciente idoso infectado pelo HIV (CLOSS VE e SCHWANKE CHA, 2012).

Pesquisas adicionais são urgentemente necessárias para entender melhor o impacto do envelhecimento no curso da infecção pelo HIV, desenvolver e implementar medidas efetivas de educação e prevenção e determinar a eficácia e segurança da terapia antirretroviral no paciente mais velho infectado pelo HIV.

\section{CONSIDERAÇÕES FINAIS}

Os dados analisados ao longo deste artigo nos possibilitou trazer as seguintes considerações: há um número crescente de idosos portadores do HIV/AIDS, sendo justificado pela eficácia do tratamento que possibilita a pessoa uma nova expectativa de vida; as pessoas idosas tendem a não perceber que é portadora do HIV, sendo menos propensas do que as mais jovens a fazer o teste do HIV; os sinais de HIV / AIDS podem ser confundidos com as dores do envelhecimento normal, o que tarda o diagnóstico correto; existem muitos tabus quando o assunto é sexualidade na terceira idade; os idosos, necessitam de maior atenção e esclarecimento no tocante a sexualidade e doenças sexualmente transmissíveis. Tais constatações apenas alertam para a necessidade de realizar novas pesquisas e aprimorar tais dados de modo a contribuir cientificamente para a conscientização da HIV/AIDS na terceira idade, possibilitando novas discussões e intervenções sociais.

\section{REFERÊNCIAS}

1. ALMEIDA T, LOURENÇO ML. Reflexões: conceitos, estereótipos e mitos acerca da velhice. Revista Brasileira de Ciências do Envelhecimento Humano, 2009; 6(2): 233-244.

2. BATISTA AFO, et al. Idosos: associação entre o conhecimento da aids, atividade sexual e condições sociodemográficas. Revista Bras. de Geriatria e Gerontologia, 2011; 14(1): 39-48.

3. BRASIL, Ministério da Saúde. Secretaria de Vigilância em Saúde. Departamento de vigilância epidemiológica. Boletim Epidemiológico - HIV AIDS, 2019; Número Especial, Dez: 1-72.

4. BRASIL. Secretária de Vigilância da Saúde - Ministério da Saúde - Programa Nacional de DST e Aids. "Aids: etiologia, clínica, diagnóstico e tratamento" Unidade de Assistência. 1999. Disponível em: http://bvsms.saude.gov.br/bvs/publicacoes/Aids_etiologia_clinica_diagnostico_ttratament.pdf. Acesso em em 12 de jan de 2020. 
5. CAMARGO CV, et al. Concepção pragmática e concepção científica dos adolescentes sobre a AIDS. Estudos em Psicologia, 2007; v.12, n.2, p. 277-284.

6. CASAES NRR. Suporte social e vivência de estigma: um estudo entre pessoas com HIV/AIDS. Dissertação (Mestrado em Psicologia) - Universidade Federal da Bahia, Faculdade de Filosofia e Ciências Humanas, Salvador, 2007; 123 p.

7. CLOSS VE, SCHWANKE CHA. A Evolução do Índice de Envelhecimento no Brasil, nas suas Regiões e Unidades Federativas no Período de 1970 a 2010. Revista Brasileira de Geriatria e Gerontologia. 2012; 15 (3): $443-458$.

8. DRIEMEIER M, et al. Vulnerabilidade à AIDS entre idosos em um centro urbano da região central do Brasil. Clinics, 2012; 67(1), 19-25.

9. FONSECA JJS. Metodologia da pesquisa científica. Fortaleza: UEC, 2002, 127 p.

10. FREITAS MRI, et al. Compreendendo a sexualidade de indivíduos portadores de HIV-1. Rev. esc. Enferm, $2000 ; 34$ (3): 258-263, 2000.

11. GARCIA GS, et al. Vulnerabilidade dos idosos frente ao HIV/aids: tendências da produção científica atual no Brasil. DST - J Bras. Doenças Sex. Transm. 2012; 24(3):183-188.

12. GIL AC. Como elaborar projetos de pesquisa - 4. ed. - São Paulo: Atlas, 2002, 176 p.

13. GUIMARÃES R, FERRAZ AF. A interface aids, estigma e identidade - algumas considerações. Revista Mineira de Enfermagem, 2002; 6(2):77-85.

14. IBGE, 2019. In: Instituto Brasileiro de Geografia e Estatística. Expectativa de vida dos brasileiros aumenta para 76,3 anos em 2018. Disponível em: https://agenciadenoticias.ibge.gov.br/agencia-noticias/2012-agencia-denoticias/noticias/26103-expectativa-de-vida-dos-brasileiros-aumenta-para-76-3-anos-em-2018. Acesso em 12 de jan de 2020.

15. MACIEL MG. Atividade física e funcionalidade do idoso. Motriz, 2010; 16 (4):1024-1032.

16. MILZ T. O que explica o aumento dos casos de HIV no Brasil? 2019. Disponível em: http://bvsms.saude.gov.br/bvs/publicacoes/Aids_etiologia_clinica_diagnostico_ttratament.pdf. Acesso em em 10 de jan de 2020.

17. MELO HMA, et al. O conhecimento sobre aids de homens idosos e adultos jovens: um estudo sobre a percepção desta doença. Ciência \& Saúde Coletiva, 2012; 17(1): 43-53.

18. MONTEIRO JFA, FIGUEIREDO MAC. Vivência profissional: subsídios à atuação em HIV/Aids. Revista Paidéia, 2009; 19(42): 67-79.

19. PARKER R, AGGLETON P. Estigma, discriminação e AIDS. Coleção ABIA: Cidadania e direitos, $2001 ; 45$ p.

20. RISMAN A. Sexualidade e terceira idade: uma visão histórico-cultural. Textos Envelhecimento., 2005; 8(1): 89-115.

21. SANTOS AFM, ASSIS M. Vulnerabilidade das idosas ao HIV/AIDS: despertar das políticas públicas e profissionais de saúde no contexto da atenção integral: revisão de literatura. Revista Brasileira de Geriatria e Gerontologia, 2011, 14(1): 147-157.

22. SILVA LC, et al. Envelhecimento e HIV/aids: contribuições da psicologia social ao ensino e pesquisa em saúde. CONVIBRA SAUDE, 2013, 1 (1): 1-12.

23. SILVA MM, et al. Caracterização epidemiológica dos casos de AIDS em pessoas com 60 anos ou mais, Pernambuco, Brasil, 1998 a 2008. Cad. Saúde Pública, Rio de Janeiro, 2013; 29(10):2131-2135.

24. SILVA VXL, et al. Considerações sobre a sexualidade dos idosos nos textos gerontológicos. Revista Brasileira de Geriatria e Gerontologia, 2009; 12(2): 295-303.

25. SMIT M, et al. Desafios futuros para o atendimento clínico de uma população idosa infectada pelo HIV: um estudo de modelagem. Lancet Infect Dis. 2015; 15: 810-818.

26. SOUZA IB, et. al. Perfil sociodemográfico de idosos com vírus da imunodeficiência humana em um estado do nordeste brasileiro. Rev. bras. geriatr. gerontol. 2019; 22(4): 1-9.

27. ROSSI SMG, et al. Impacto da terapia antirretroviral conforme diferentes consensos de tratamento da Aids no Brasil. Rev Panam Salud Publica, 2012, 32(2): 117-123.

28. UNAIDS, 2020. In: Estatísticas. Brasil. Disponível em: https://unaids.org.br/estatisticas/ Acesso em 18 de nov de 2020. 